\title{
A BETTER CALCULUS OF MOVING SURFACES
}

\author{
PAVEL GRINFELD
}

Communicated by Gregory L. Naber

Abstract. We introduce $\dot{\nabla}$, a new invariant time derivative with respect to a moving surface that is a modification of the classical $\delta / \delta$-derivative. The new operator offers significant advantages over its predecessor. In particular, it produces zero when applied to the surface metric tensors $S_{\alpha \beta}$ and $S^{\alpha \beta}$ and therefore permits free juggling of surface indices in the calculus of moving surfaces identities. As a result, the table of essential differential relationships is cut in half. To illustrate the utility of the operator, we present a calculus of moving surfaces proof of the Gauss-Bonnet theorem for smooth closed two dimensional hypersurfaces.

\section{Introductory Remarks}

The $\delta / \delta t$-derivative has been the cornerstone of the calculus of moving surfaces, an extension of tensor calculus to deforming manifolds. Here, we introduce a new invariant time derivative $\dot{\nabla}$ which, because of the several conveniences that it offers over the $\delta / \delta t$-derivative, will replace it in the future. As a demonstration of the new operator's utility, we will give a proof of a special case of the GaussBonnet theorem by showing that the integral of Gaussian curvature over a closed surface remains unchanged under smooth surface evolutions that preserve its topology. Naturally, the Gauss-Bonnet theorem, which is a statement about the intrinsic geometry of a surface, is celebrated for its much deeper topological meaning. On the other hand, the calculus of moving surfaces (CMS) is extrinsic by its very nature. Nevertheless, the proof represents an effective application of the calculus of moving surfaces to a problem in which there are no moving surfaces.

In differential geometry on stationary manifolds, invariance is achieved by introducing the covariant derivative $\nabla_{\alpha}$ that replaces the partial derivative $\partial / \partial S^{\alpha}$ [13], [8], [7], [9]. On moving surfaces, the calculus of moving surfaces plays a role analogous to that of tensor calculus on stationary surfaces. The central operator in the calculus of moving surfaces is the $\delta / \delta t$-derivative, which is the analogue of the covariant derivative $\nabla_{\alpha}$. The calculus of moving surfaces has proven effective at analyzing problems in dynamics [2], [5], boundary perturbation [4], shape optimization [3] and, as seen in this paper, intrinsic problems on stationary surfaces. 
The $\delta / \delta t$-derivative possessed most of the desirable properties of the covariant derivative, the central of which is preserving the tensor property of its operands. On the other hand, an application of the $\delta / \delta t$-derivative applied to the metric tensors $S_{\alpha \beta}$ and $S^{\alpha \beta}$ does not produce zero. As a result, surface indices cannot be juggled freely in CMS identities and therefore the table of differentiation must contain several rules for each object with surface indices. For example, for the curvature tensor $B_{\beta}^{\alpha}$, the CMS gives three rules depending on the flavor of the indices

$$
\begin{aligned}
\frac{\delta B^{\alpha \beta}}{\delta t} & =\nabla_{\beta} \nabla^{\alpha} C+3 C B_{\gamma}^{\alpha} B_{\beta}^{\gamma} \\
\frac{\delta B_{\beta}^{\alpha}}{\delta t} & =\nabla_{\beta} \nabla^{\alpha} C+C B_{\gamma}^{\alpha} B_{\beta}^{\gamma} \\
\frac{\delta B_{\alpha \beta}}{\delta t} & =\nabla_{\beta} \nabla^{\alpha} C-C B_{\gamma}^{\alpha} B_{\beta}^{\gamma} .
\end{aligned}
$$

The new operator $\dot{\nabla}$ proposed here overcomes these problems. It produces zero when applied to the metric tensors and therefore permits free juggling of surface indices. As a result, virtually all identities of the calculus of moving surfaces are simplified and their overall number is reduced. For example, the three curvature rules (1)-(3) are replaced by a single one (24). Furthermore, when applied to the Levi-Civita symbols $\varepsilon_{\alpha \beta}$ and $\varepsilon^{\alpha \beta}$, the $\dot{\nabla}$ operator operator also produces zero. Thus, the $\dot{\nabla}$ operator possesses all of the desirable properties of the covariant derivative $\nabla_{\alpha}$.

The original prototype of the $\delta / \delta t$-derivative was proposed by Hadamard, [6]. Hadamard gave the geometric definition illustrated in Fig. 1 and derived its analytical represntation Hadamard's definition applied to geometric invariants. The extension to tensor operands was accomplished over the years by several authors, including Thomas [10], [11], and Truesdell and Toupin [12]. A definition applicable to arbitrary tensors was finally given by Grinfeld [1]. This definition turned the moving surfaces framework into an algebraically completely calculus and serves as a basis for the new operator given here.

\section{Notation}

Suppose that the ambient three dimensional space is referred to Cartesian coordinates $Z^{i}(i=1,2,3)$ and that the embedded manifold $S$ is referred to coordinates $S^{\alpha}(\alpha=1,2)$. The smooth evolution of the manifold is given by

$$
Z^{i}=Z^{i}(t, S)
$$




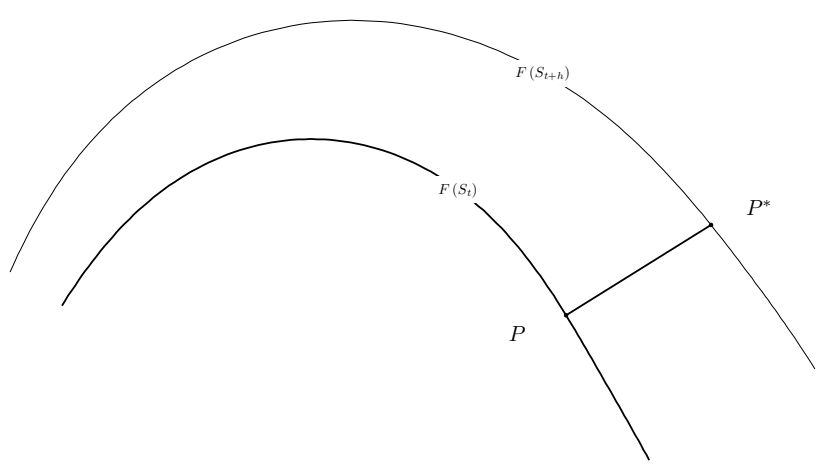

Figure 1. Illustration of Hadamard's geometric definition of the $\delta / \delta t$ derivative for scalar fields. If $F$ a scalar field defined on a deforming surface $S_{t}, \delta F / \delta t$ at a point $P$ is defined as the limit as $h \rightarrow 0$ of $\left(F\left(P^{*}\right)-F(P)\right) / h$ where $P^{*}$ is the point on $S_{t+h}$ that intersects the normal to $S_{t}$ at the point $P$. The $\delta / \delta t$-derivative and the new $\dot{\nabla}$ operator produce the same result when applied to invariants.

where we follow the convention of dropping the tensor indices of function arguments. The coordinate velocity $V^{i}$ is defined by

$$
V^{i}(t, S)=\frac{\partial Z^{i}(t, S)}{\partial t} .
$$

Let $N^{i}$ be contravariant components of the normal to the surface $S$. Then the quantity

$$
C=V_{i} N^{i}
$$

is the Hadamard velocity of the interface $S$. It is invariant with respect to coordinate changes in the ambient space and on the surface $S$.

We now list the essential differential objects in the ambient space and on the surface [13], [8], [7], [9]. In the ambient space, $Z_{i j}$ is the covariant metric tensor, $Z^{i j}$ is the contravariant metric tensor, $\varepsilon^{i j k}$ and $\varepsilon_{i j k}$ are the Levi-Civita symbols, $\nabla_{i}$ and $\nabla^{i}$ and the covariant and the contravariant metric tensors, $\Gamma_{j k}^{i}$ is the Christoffel symbol, and $R_{i j k l}$ is the Riemann-Christoffel tensor. On the surface, $Z_{\alpha}^{i}$ is the shift tensor, $S_{\alpha \beta}$ is the covariant metric tensor, $S^{\alpha \beta}$ is the contravariant metric tensor, $B_{\alpha \beta}$ is the curvature tensor, $\varepsilon^{\alpha \beta}$ and $\varepsilon_{\alpha \beta}$ are the Levi-Civita symbols, $\nabla_{\alpha}$ and $\nabla^{\alpha}$ and the covariant and contravariant surface derivatives, $\Gamma_{\beta \gamma}^{\alpha}$ is the Christoffel symbol and $R_{\alpha \beta \gamma \delta}$ is the Riemann-Christoffel tensor. The Riemann-Christoffel tensor is skew-symmetric in the first and the second pairs of indices and can be represented as

$$
R_{\alpha \beta \gamma \delta}=K \varepsilon_{\alpha \beta} \varepsilon_{\gamma \delta}
$$


where $K$ is Gaussian curvature. Multiplying both sides by $\varepsilon^{\alpha \beta} \varepsilon^{\gamma \delta}$ yields an explicit expression for $K$

$$
K=\frac{1}{4} \varepsilon^{\alpha \beta} \varepsilon^{\gamma \delta} R_{\alpha \beta \gamma \delta} .
$$

The ambient and the surface Riemann-Christoffel tensors are connected by the formula

$$
R_{\alpha \beta \gamma \delta}=R_{i j k l} Z_{\alpha}^{i} Z_{\beta}^{j} Z_{\gamma}^{k} Z_{\delta}^{l}+B_{\alpha \gamma} B_{\beta \delta}-B_{\alpha \delta} B_{\beta \gamma} .
$$

When the ambient space is Euclidean, this formula reduces to

$$
R_{\alpha \beta \gamma \delta}=B_{\alpha \gamma} B_{\beta \delta}-B_{\alpha \delta} B_{\beta \gamma}
$$

known as Gauss's Theorema Egregium. The combination $B_{\alpha \gamma} B_{\beta \delta}-B_{\alpha \delta} B_{\beta \gamma}$ can be expressed in terms of the Levi-Civita symbols

$$
B_{\alpha \gamma} B_{\beta \delta}-B_{\alpha \delta} B_{\beta \gamma}=B \varepsilon_{\alpha \beta} \varepsilon_{\beta \gamma}
$$

where $B$ is the determinant of the curvature tensor $B_{\beta}^{\alpha}$ with a raised index

$$
B=\frac{1}{2} \delta_{\gamma \delta}^{\alpha \beta} B_{\alpha}^{\gamma} B_{\delta}^{\beta}
$$

The delta system $\delta_{\gamma \delta}^{\alpha \beta}$ is defined in terms of the Levi-Civita symbols

$$
\delta_{\gamma \delta}^{\alpha \beta}=\varepsilon^{\alpha \beta} \varepsilon_{\gamma \delta}
$$

and can be represented in terms of the Kronecker delta

$$
\delta_{\gamma \delta}^{\alpha \beta}=\delta_{\gamma}^{\alpha} \delta_{\delta}^{\beta}-\delta_{\gamma}^{\beta} \delta_{\delta}^{\alpha} .
$$

From equations (7), (10) and (11), we conclude that Gaussian curvature $K$ of a surface embedded in a Euclidean space equals $B$

$$
K=B .
$$

We will employ the calculus of moving surfaces to show that

$$
\frac{\mathrm{d}}{\mathrm{d} t} \int_{S} K \mathrm{~d} S=0 .
$$

In words, the total curvature of a closed surfaces in unchanged under smooth deformations. 


\section{Definition of the Invariant Time Derivative $\dot{\nabla}$}

Denote the projection of the coordinate velocity $V^{i}$ onto the surface by $V^{\alpha}$

$$
V^{\alpha}=V^{i} Z_{i}^{\alpha}
$$

and define the object $\dot{\Gamma}_{\beta}^{\alpha}$ according to

$$
\dot{\Gamma}_{\beta}^{\alpha}=\nabla_{\beta} V^{\alpha}-C B_{\beta}^{\alpha}
$$

Suppose that $T_{j \beta}^{i \alpha}$ is a tensor with a representative collection of indices defined on the surface $S$. Then the invariant derivative $\dot{\nabla} T_{j \beta}^{i \alpha}$ is defined according to

$$
\dot{\nabla} T_{j \beta}^{i \alpha}=\frac{\partial T_{j \beta}^{i \alpha}}{\partial t}-V^{\gamma} \nabla_{\gamma} T_{j \beta}^{i \alpha}+V^{k} \Gamma_{k m}^{i} T_{j \beta}^{m \alpha}-V^{k} \Gamma_{k j}^{m} T_{m \beta}^{i \alpha}+\Gamma_{\omega}^{\alpha} T_{j \beta}^{i \omega}-\Gamma_{\beta}^{\omega} T_{j \omega}^{i \alpha} .
$$

Thus, the invariant time derivative $\dot{\nabla}$ differs from the $\delta / \delta t$-derivative in its treatment of surface indices.

The single most essential advantage that $\dot{\nabla}$ offers over the $\delta / \delta t$-derivative is that it eliminates the surface metric tensors

$$
\dot{\nabla} S_{\alpha \beta}=0, \quad \dot{\nabla} S^{\alpha \beta}=0 .
$$

It also eliminates the surface Levi-Civita symbol

$$
\dot{\nabla} \varepsilon_{\alpha \beta}=0, \quad \dot{\nabla} \varepsilon^{\alpha \beta}=0 .
$$

The former property allows for free index juggling across the operator. This leaves us with only three essential identities that a practitioner of this calculus ought to memorize

$$
\begin{aligned}
\dot{\nabla} Z_{\alpha}^{i} & =N^{i} \nabla_{\alpha} C \\
\dot{\nabla} N^{i} & =-Z_{\alpha}^{i} \nabla^{\alpha} C \\
\dot{\nabla} B_{\beta}^{\alpha} & =\nabla^{\alpha} \nabla_{\beta} C+C B_{\gamma}^{\alpha} B_{\beta}^{\gamma} .
\end{aligned}
$$

The remaining properties of the $\dot{\nabla}$ operator are identical to those of the $\delta / \delta t$ derivative. The $\dot{\nabla}$ operator satisfies the product rule and the chain rule that governs the differentiation of surface restrictions of ambient tensor fields

$$
\dot{\nabla} T_{j}^{i}=\frac{\partial T_{j}^{i}}{\partial t}+C N^{k} \nabla_{k} T_{j}^{i}
$$




\section{Gauss-Bonnet Theorem in Euclidean spaces}

We next calculate the derivative $\dot{\nabla} K$ of Gaussian curvature $K$ for two-dimensional surfaces $S$ embedded in a Euclidean space. Since $\dot{\nabla}$ eliminates the Levi-Civita symbols $\varepsilon_{\alpha \beta}$ and $\varepsilon^{\alpha \beta}$ it also eliminates $\delta_{\gamma \delta}^{\alpha \beta}=\varepsilon^{\alpha \beta} \varepsilon_{\gamma \delta}$. Therefore

$$
\dot{\nabla} K=\frac{1}{2} \delta_{\gamma \delta}^{\alpha \beta} \dot{\nabla} B_{\alpha}^{\gamma} B_{\beta}^{\delta}+\frac{1}{2} \delta_{\alpha \beta}^{\gamma \delta} B_{\alpha}^{\gamma} \dot{\nabla} B_{\beta}^{\delta} .
$$

According to equation (24) we find that

$$
\frac{1}{2} \delta_{\gamma \delta}^{\alpha \beta} \dot{\nabla} B_{\alpha}^{\gamma} B_{\beta}^{\delta}=\frac{1}{2} \delta_{\gamma \delta}^{\alpha \beta}\left(\nabla_{\alpha} \nabla^{\gamma} C+C B_{\alpha}^{\omega} B_{\omega}^{\gamma}\right) B_{\beta}^{\delta} .
$$

Since the combination $\delta_{\gamma \delta}^{\alpha \beta} B_{\alpha}^{\omega} B_{\beta}^{\delta}$ is skew-symmetric in $\omega$ and $\delta$, we have

$$
\delta_{\alpha \beta}^{\gamma \delta} B_{\omega}^{\alpha} B_{\delta}^{\beta}=B \delta_{\omega \delta}^{\gamma \delta}=B \delta_{\omega}^{\gamma} .
$$

Therefore

$$
\frac{1}{2} \delta_{\gamma \delta}^{\alpha \beta} \dot{\nabla} B_{\alpha}^{\gamma} B_{\beta}^{\delta}=\frac{1}{2} \nabla_{\alpha} \nabla^{\alpha} C B_{\beta}^{\beta}-\frac{1}{2} \nabla_{\alpha} \nabla^{\beta} C B_{\beta}^{\alpha}+\frac{1}{2} C B B_{\alpha}^{\alpha} .
$$

The two terms in (26) are equivalent and therefore

$$
\dot{\nabla} K=\nabla_{\alpha} \nabla^{\alpha} C B_{\beta}^{\beta}-\nabla_{\alpha} \nabla^{\beta} C B_{\beta}^{\alpha}+C K B_{\alpha}^{\alpha} .
$$

The rate of change of integrals over closed surfaces is given by the formula

$$
\frac{\mathrm{d}}{\mathrm{d} t} \int_{S} F \mathrm{~d} S=\int_{S}\left(\dot{\nabla} F-C B_{\alpha}^{\alpha} F\right) \mathrm{d} S .
$$

Therefore

$$
\frac{\mathrm{d}}{\mathrm{d} t} \int_{S} K \mathrm{~d} S=\int_{S}\left(\nabla_{\alpha} \nabla^{\alpha} C B_{\beta}^{\beta}-\nabla_{\alpha} \nabla^{\beta} C B_{\beta}^{\alpha}\right) \mathrm{d} S .
$$

Since $S$ does not have a boundary, then by Gauss's theorem

$$
\frac{\mathrm{d}}{\mathrm{d} t} \int_{S} K \mathrm{~d} S=\int_{S}\left(-\nabla_{\alpha} C \nabla^{\alpha} B_{\beta}^{\beta}+\nabla_{\alpha} C \nabla^{\beta} B_{\beta}^{\alpha}\right) \mathrm{d} S .
$$

However, according to Codazzi relations the object $\nabla_{\alpha} B_{\beta \gamma}$ is symmetric in $\alpha$ and $\beta$

$$
\nabla_{\alpha} B_{\beta \gamma}=\nabla_{\beta} B_{\alpha \gamma} .
$$

By raising $\alpha$ and $\beta$ in this equation and contracting, we conclude that the integral in (33) vanishes, which proves (16) in Euclidean spaces. 


\section{The Gauss-Bonnet Theorem in Riemann Spaces}

Equations (24) and (34) are not valid in Riemann spaces and we will now give their proper generalizations. The derivative $\dot{\nabla} B_{\alpha \beta}$ of the curvature tensor $B_{\alpha \beta}$ is given by

$$
\dot{\nabla} B_{\alpha \beta}=\nabla_{\alpha} \nabla_{\beta} C+C B_{\alpha}^{\gamma} B_{\gamma \beta}+C R_{i j k l} N^{i} Z_{\beta}^{j} N^{k} Z_{\alpha}^{l} .
$$

The Codazzi relations read

$$
\nabla_{\alpha} B_{\beta \gamma}-\nabla_{\beta} B_{\alpha \gamma}=-R_{i j k l} Z_{\alpha}^{i} Z_{\beta}^{j} Z_{\gamma}^{k} N^{l}
$$

Finally, the Gaussian curvature $K$ no longer equals the determinant $B$, but rather related to it by the formula

$$
K=B+\frac{1}{2} R_{i}^{i}+R_{i j} N^{i} N^{j} .
$$

Adapting the Euclidean derivation of $\dot{\nabla} B$ to Riemann spaces, we find

$$
\begin{aligned}
\dot{\nabla} B=\nabla_{\alpha} \nabla^{\alpha} C B_{\beta}^{\beta}-\nabla_{\alpha} & \nabla^{\beta} C B_{\beta}^{\alpha}+2 C B B_{\alpha}^{\alpha}-C K B_{\alpha}^{\alpha} \\
& +C R_{i}^{i} B_{\alpha}^{\alpha}-C R_{i j} N^{i} N^{j} B_{\alpha}^{\alpha}-C R_{i j} Z^{i \beta} Z_{\alpha}^{j} B_{\beta}^{\alpha}
\end{aligned}
$$

and, by applying the chain rule, we find from equation (37) we find

$$
\dot{\nabla} K=\dot{\nabla} B+\frac{1}{2} C N^{k} \nabla_{k} R_{i}^{i}-C \nabla_{k} R_{i j} N^{i} N^{j} N^{k}+2 R_{i j} Z_{\alpha}^{i} \nabla^{\alpha} C N^{j} .
$$

From the second Bianchi identity, we find

$$
\nabla_{k} R_{i}^{i}=2 \nabla_{i} R_{k}^{i}
$$

Thus

$$
\dot{\nabla} K=\dot{\nabla} B+C \nabla_{i} R_{k}^{i} N^{k}-C \nabla_{k} R_{i j} N^{i} N^{j} N^{k}+2 R_{i j} Z_{\alpha}^{i} \nabla^{\alpha} C N^{j} .
$$

We now sketch the rest of the derivation. The expression for $\dot{\nabla} K$ includes three terms that contain the surface derivative $\nabla_{\alpha} C$ of $C: \nabla_{\alpha} \nabla^{\alpha} C B_{\beta}^{\beta}, \nabla_{\alpha} \nabla^{\beta} C B_{\beta}^{\alpha}$, and $2 R_{i j} Z_{\alpha}^{i} \nabla^{\alpha} C N^{j}$. These terms are analyzed by Gauss's theorem. The first two terms produce an expression that can be advanced by the Codazzi relations (36) followed by a second application of Gauss's theorem. We ultimately arrive the integral

$$
\frac{\mathrm{d}}{\mathrm{d} t} \int_{S} K \mathrm{~d} S=\int_{S} 2 C\left(B-K+\frac{1}{2} R_{i}^{i}-R_{i j} N^{i} N^{j}\right) B_{\alpha}^{\alpha} \mathrm{d} S .
$$

The integrand vanishes identically by equation (37) and we have therefore demonstrated (16) in Riemann spaces. 


\section{Fluid Film Equations}

The fluid film equations, first introduced in [2], are formulated in terms of the $\delta / \delta t$-derivative. The last equation in the system, governs the evolution of the contravariant components $V^{\alpha}$ of the tangential velocity field and features the term $\delta V^{\alpha} / \delta t$ on the left hand side. Therefore, the corresponding equation for the evolution of the covariant components $V_{\alpha}$ is different. The new operator $\dot{\nabla}$ eliminates this incovenience and yields a system that is valid for covariant and contravariant components. In the new form, the system reads

$$
\begin{aligned}
\dot{\nabla} \rho+\nabla_{\alpha}\left(\rho V^{\alpha}\right) & =\rho C B_{\alpha}^{\alpha} \\
\rho\left(\dot{\nabla} C+2 V^{\alpha} \nabla_{\alpha} C+B_{\beta}^{\alpha} V_{\alpha} V^{\beta}\right) & =-\rho^{2} e_{\rho} B_{\alpha}^{\alpha} \\
\rho\left(\dot{\nabla} V_{\alpha}+V_{\beta} \nabla^{\beta} V_{\alpha}-C \nabla_{\alpha} C-C V_{\beta} B_{\alpha}^{\beta}\right) & =-\nabla_{\alpha}\left(\rho^{2} e_{\rho}\right) .
\end{aligned}
$$

Note we in fact mixed the covariant and contraviant components $V_{\alpha}$ and $V^{\alpha}$ which we can now do more freely than before.

\section{Conclusion}

We introduced a new differential operator $\dot{\nabla}$ for the calculus of moving surfaces. The definition is given in equations (17)-(19) and yields the highly desirable property that the derivative of the metric tensors $S_{\alpha \beta}$ and $S^{\alpha \beta}$ vanishes. As a result, surface indices can be juggled freely in tensor identities involving the operator. This was not the case for the $\delta / \delta t$-derivative. Therefore, the new operator $\dot{\nabla}$ simplifies the calculations and cuts in half the table of essential relationships. As a demonstration of the new operator, we gave a proof of a special case of the GaussBonnet theorem which shows that, in addition to dynamic problems, boundary perturbation problems and shape optimization problems, the calculus of moving surfaces finds applications in problems that do not have moving surfaces.

\section{References}

[1] Grinfeld M., Thermodynamic Methods in the Theory of Heterogeneous Systems, Longman, New York 1991.

[2] Grinfeld P., Exact Nonlinear Equations for Fluid Films and Proper Adaptations of Conservation Theorems From Classical Hydrodynamics, J. Geom. Symmetry. Phys. 16 (2009) 1-21. 
[3] Grinfeld P., Shape Optimization and Electron Bubbles, Num. Func. Anal. Opt. 30 (2009) 689-710.

[4] Grinfeld P. and Strang G., Laplace Eigenvalues on Regular Polygons: A Series in 1/N, Submitted to Trans. AMS, 2009.

[5] Grinfeld P. and Wisdom J., The Effect of Phase Transformations at the Inner Core Boundary on the Slichter Modes, Phys. Earth Plan. Int. 178 (2010) 183-188.

[6] Hadamard J., Leçons sur la propagation des ondes et les équations de l'hydrodynamique, Hermann, Paris 1903.

[7] Levi-Civita T., The Absolute Differential Calculus (Calculus of Tensors). Dover, New York 1977.

[8] McConnell A., Applications of Tensor Analysis, Dover, New York 1957.

[9] Synge J. and Schild A., Tensor Calculus, Dover, New York 1978.

[10] Thomas T., Extended Compatibility Conditions for the Study of Surface of Discontinuity in Continuum Mechanics, J. Math. Mech. 6 (1957) 311-322.

[11] Thomas T., Plastic Flow and Fracture in Solids, Academic Press, New York 1961.

[12] Truesdell C. and Toupin R., The Classical Field Theories, Springer, Berlin 1960.

[13] Weyl H., Space, Time, Matter, Dover, New York 1952.

Pavel Grinfeld

Department of Mathematics

271 Korman

Drexel University

Philadelphia, PA 19105

USA

E-mail address: pg@freeboundaries.com 